\title{
Wireless Sensor Network Based Remote Environmental Conditions Monitoring system with GPRS
}

\author{
Shaik.Rehana Sutana ${ }^{1}$, P.Bala Krishan ${ }^{1}$, K.Gouthami ${ }^{1}$ and Tai-hoon Kim ${ }^{2}$ \\ ${ }^{I}$ Department of Electronics and Communication Engineering, \\ Vignan's Nirula Institute of Technology and Science for Women, \\ Palakaluru, Guntur, AP, India \\ Skrehana227@gmail.com \\ ${ }^{2}$ Department of Convergence Security, Sungshin Women's University, \\ 249-1, Dongseon-dong 3-ga, Seoul, 136-742, Korea \\ taihoonn@daum.net \\ (Corresponding Author)
}

\begin{abstract}
Wireless sensor networks (WSN) are the network having number of sensors to transfer data from source to destination. In the internet point of view the connect number, sampling rate and signal types of sensors are generally restricted by the device. In the network, there are no of sensors are present and each sensor gives sensing when the data transfer occurs at that node. In the modern scenario wireless networks contains sensors as well as actuators. ZigBee is newly developed technology that works on IEEE standard 802.15.4, which can be used in the wireless sensor network (WSN). The low data rates, low power consumption, low cost are main features of ZigBee. To improve the performance of ZigBee a new method is proposed to reconfigurable smart sensor interface for industrial WSN in IoT environment, in which ARM7 is adopted as the core controller. Performance will show the effects of proposed system.
\end{abstract}

Keywords: ARM-7 Board, Zigbee Module, Smart Phone, Sensors, Controlled Devices, WIFI

\section{Introduction}

Wireless sensor network is an innovation for extensive variety of wireless environments. As of late more research work has been done in bearing to develop wireless network that works on low power, low data rate, low cost personal area network. Large numbers of companies are developing WSNs for smart home, smart farm, smart hospital for patient monitoring, for traffic monitoring in VANET, fire monitoring in smart cities. The significance and application has been expanded by the recent delivery of the IEEE 802.15.4 standard and the up next ZigBee standard. The ZigBee Alliance has grown exceptionally minimal cost, very low-power consumption, wireless communications standard for system and application layer to satisfy the demand of automation and remote control applications. IEEE 802.15.4 committee started working on a low data rate standard a short while later for physical and MAC sub layer. Then the ZigBee Alliance and the IEEE decided to join forces and ZigBee is the commercial name for this technology. 


\section{The Hardware System}

\subsection{Micro Controller}

This section forms the control unit of the whole project. This area fundamentally comprises of a Microcontroller with its related hardware like Crystal with capacitors, Reset circuitry, Pull up resistors (if necessary). The Microcontroller structures the heart of the undertaking on the grounds that it controls the devices being interfaced and corresponds with the devices as per the system being written.

\subsection{ARM7TDMI}

ARM stands for Advanced RISC Machines, it is the name of a class of processors, and is the name of a kind technology too. The RISC rule set, and related decode mechanism are much easier than those of Complex Instruction Set Computer (CISC) outlines.

\subsection{Liquid-Crystal Display}

LCD stands for Liquid-crystal display which is a flat panel display which uses the properties of light modulation liquid crystals. Liquid crystals don't radiate light directly. LCDs are accessible to show arbitrary images or fixed images which can be displayed or hidden, such as preset words, digits, and 7-segment displays as in a digital clock. They utilize the same fundamental innovation, aside from that arbitrary pictures are comprised of a substantial number of little pixels, while different presentations have bigger components.

\subsection{Thermistor}

Thermistors are a temperature sensing devise. It is used to sense the temperature. In this project by depends on the value of temperature the exhaust fan will run.

\subsection{LDR}

The LDR is used to measure the light intensity. According to that the sensed information is given to Microcontroller which will send to monitoring section through ZigBee.

\section{Design of Proposed Hardware System}

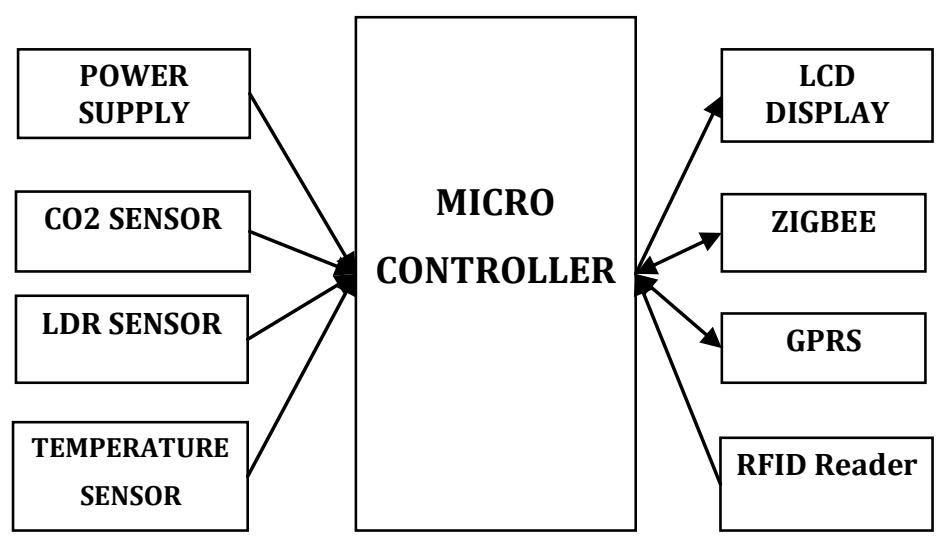

Figure 1. Transmitting Section 


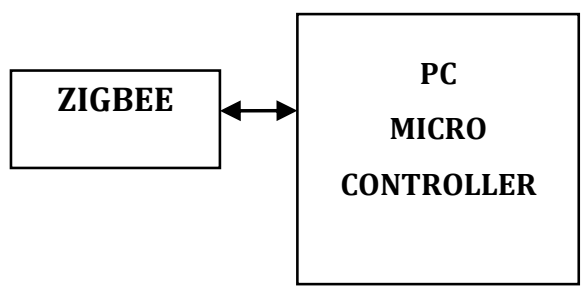

Figure 2. Monitoring Section

The design of entire system consisted of two part which are hardware and software. The hardware is designed by the rules of embedded system, and the steps of software consisted of three parts. Zigbee based wireless technology which comprises of transmitter at the server and receiver at control board. Data received at the recipient will be send to the PC through Zigbee. So the users living at home with web can see the received information. The framework utilizes a smaller hardware fabricated around LPC2148 (ARM7) microcontroller Programs are produced in Embedded C.

\section{Board Hardware Resources Features}

\subsection{Thermistor}

For the measurement of the temperature the most commonly used device is Thermistors. Resisters work according to the temperature the changes dynamically. While for the greater part of the metals the resistance increases with temperature, the thermistors react negatively to the temperature and their resistance decreases with the increment in temperature. Since the resistance of thermistors is subject to the temperature, they can be associated in the electrical circuit to quantify the temperature of the body.

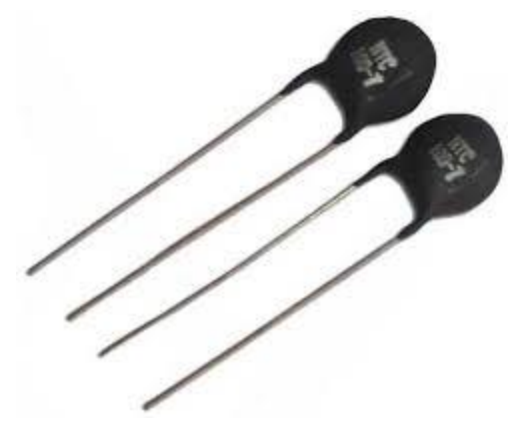

Figure 3. Thermistors

\subsection{LDR}

LDRs or Light Dependent Resistors are extremely valuable particularly in light/dull sensor circuits. Typically the resistance of a LDR is high, in some cases as high as $1000000 \mathrm{ohms}$, however when they are lit up with light resistance drops drastically.

The movement inverse demonstrates that when the light is turned on, the resistance of the LDR falls, permitting current to go through it. This is a sample of a light sensor circuit: When the light level is low the resistance of the LDR is high. This keeps current from streaming to the base of the transistors. Thus the LED does not light. Be that as it may, when light sparkles onto the LDR its resistance falls and current streams into the base of the first transistor and afterward the second transistor. The LED lights on. The preset resistor can be turned up or down to build or diminishing resistance; along these lines it can make the circuit pretty much delicate. 


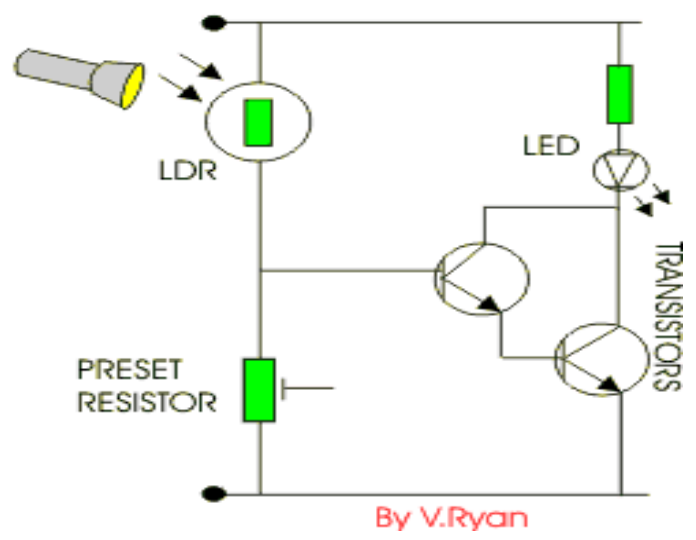

Figure 4. Light Dependent Resistors

\subsection{CO2 Sensor}

A carbon dioxide sensor or $\mathrm{CO} 2$ sensor is an instrument for the estimation of carbon dioxide gas. The most well-known standards for $\mathrm{CO} 2$ sensors are infrared gas sensors and synthetic gas sensors. Measuring carbon dioxide is critical in observing indoor air quality.

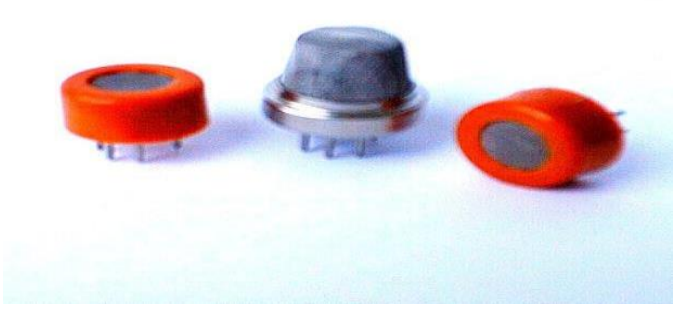

Figure 5. Co2 Sensors

\subsection{ZIGBEE}

Zigbee modules highlight a UART interface, which permits any microcontroller or microchip to instantly utilize the services of the Zigbee protocol. Each of the a Zigbee equipment designer needs to do in this as is guarantee that the host's serial port logic levels are perfect with the XBee's 2.8-to 3.4-V rationale levels. The logic level change can be performed utilizing either a standard RS-232 IC or rationale level interpreters, for example, the 74LVTH125 when the host is straightforwardly joined with the XBee UART. Data is presented to the X-Bee module through its DIN pin, and it must be in the asynchronous serial format, which consists of a start bit, 8 data bits, and a stop bit. Because the input data goes directly into the input of a UART within the X-Bee module, no bit inversions are necessary within the asynchronous serial data stream. All of the required timing and parity checking is automatically taken care of by the X-Bee's UART.

\subsection{GPRS}

General Packet Radio Service (GPRS) is a bit information innovation that permits GSM operators to launch wireless data services, for example, email and Internet access. Accordingly, GPRS gives operators the capacity to utilize information to drive extra income. GPRS is frequently called a $2.5 \mathrm{G}$ innovation in light of the fact that it is a GSM administrator's initial move toward third era $(3 \mathrm{G})$ and an initial phase in remote 
information administrations Although GPRS is an information just innovation, it aides enhance GSM voice limit.

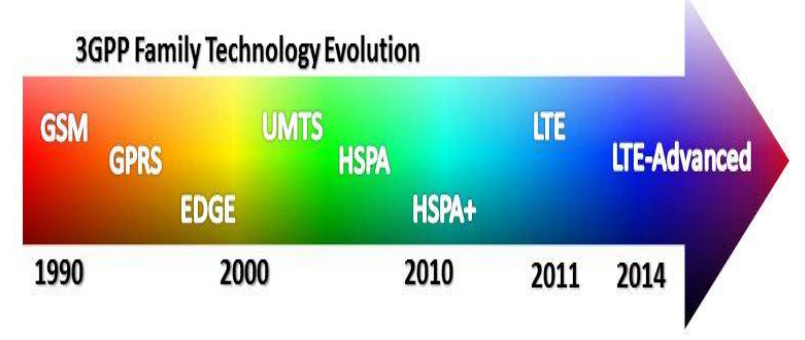

Figure 6. GPP Technology Evolution

At the point when an administrator conveys GPRS stands for General Packet Radio Service. It is a medium between GSM and $3 \mathrm{G}$ mobiles. There two types of GPRS namely $2 \mathrm{G}$ and $3 \mathrm{G}$. The transmission rate is from $9.6 \mathrm{Kbits}$ to $115 \mathrm{~K}$ bits. It is mainly used to transmission of data between mobiles. GPRS additionally permits clients to keep up an information session while noting a telephone call, which is an exceptional and selective component to GSM. GPRS likewise gives a dependably on information association, so clients don't need to sign on every time they need information access. The parcel structural engineering additionally implies that clients pay just for the information itself as opposed to for the broadcast appointment used to set up an association and download data.

GPRS is the most generally supported packet-data wireless technology in the world. Like GSM, GPRS supports universal roaming so clients can get to data services whether they are at home or abroad. At the point when clients go to zones that have not yet been moved up to GPRS, regardless they can get to numerous information administrations by means of circuit-exchanged GSM.

\subsection{RFID}

Before RFID can be seen totally, it is vital to see how Radio Frequency communication occurs. RF (Radio Frequency) correspondence happens by the transference of information over electromagnetic waves. By creating a particular electromagnetic wave at the source, its impact can be seen at the beneficiary a long way from the source, which then distinguishes it and therefore the data.

In a RFID framework, the RFID label which contains the labeled information of the item produces a sign containing the individual data which is perused by the RFID per user, which then may pass this data to a processor for preparing the acquired data for that specific application.

Along these lines, a RFID System can be visualized as the entirety of the accompanying three parts:

- RFID tag or transponder

- RFID reader or handset

- Data processing subsystem 

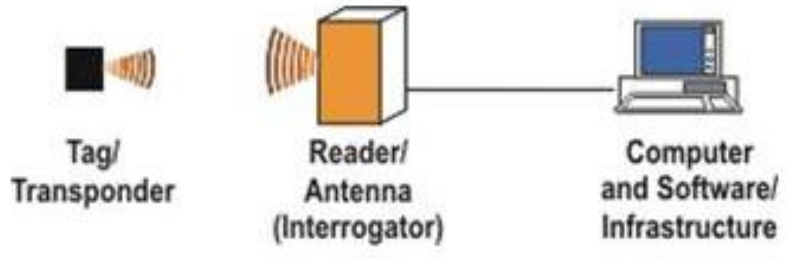

Figure 7. RFID

A RFID tag is made out of a radio wire, a remote transducer and a typifying material. These labels can be either dynamic or latent. While the dynamic labels have on-chip power, detached labels utilize the force actuated by the attractive field of the RFID per user. In this manner aloof labels are less expensive yet with lower extent $(<10 \mathrm{mts})$ and more sensitive to administrative and natural limitations, when contrasted with dynamic tags.

A RFID reader comprises of a reception apparatus, handset and decoder, which sends intermittent signs to ask about any tag in region. On getting any sign from a label it goes on that data to the information processor. The information preparing subsystem gives the method for handling and putting away the information.

\section{Conclusion}

This design is based on ZigBee and GPRS technology. The main purpose of this paper is to control the industrial appliance remotely from monitoring section. The host can know information from anywhere as all the information is posted in web page. The host controls according to the condition given by the host control that particular device. The important aim of this project is to detect the person (or) any damage of the industrial equipment and also can monitor results in web pages continuously.

\section{References}

[1] Texas Instruments, Inc., “OMAP3 Platform”, Technical report, Texas Instruments. http://www.ti.com/lit/ml/swpt024b/swpt024b.pdf, 2009.

[2] Texas Instruments, Inc., "OMAP4 Platform", Technical report, Texas Instruments. http://www.ti.com/lit/ml/swpt034b/swpt034b.pdf, 2011.

[3] Qualcomm, Inc., "Snapdragon", technical report, Qualcomm.

[4] http://www.qualcomm.com/media/documents/snapdragons4-processors-system-chip-solutions-newmobile-age, 2011.

[5] Sha, Lui, R. Rajkumar and J.P. Lehoczky, "Priority inheritance protocols: An approach to real-time synchronization", IEEE Transactions on Computers, Vol. 39, No. 9, (1990), pp. 1175-1185.

[6] Baker and P. Theodore, "A stack-based resource allocation policy for real time processes", $11^{\text {th }}$ RealTime Systems Symposium, 1990. pp. 191-200.

[7] Gai, Paolo, L. Abeni and G. Buttazzo, "Multiprocessor DSP scheduling in system-on-a-chip architectures", $14^{\text {th }}$ Euromicro Conference on Real-Time Systems, 2002 pp.231-238.

[8] Kim, Kwangsik, D. Kim and C. Park, "Real-time scheduling in heterogeneous dual-core architectures", $12^{\text {th }}$ International Conference on Parallel and Distributed Systems, ICPADS 2006, Vol. 2, pp. 6.

[9] Kato, Shinpei, K. Lakshmanan, R. Rajkumar and Y. Ishikawa, "Time Graph: GPU scheduling for realtime multi-tasking environments", USENIX ATC, pp.17-30.

[10] Kato, Shinpei, K. Lakshmanan, Y. Ishikawa and R. Rajkumar, "Resource sharing in GPU-accelerated windowing systems", $17^{\text {th }}$ IEEE Real-Time and Embedded Technology and Applications Symposium (RTAS), pp. 191-200.

[11] Saewong, Saowanee and R.R. Rajkumar, "Cooperative scheduling of multiple resources", $20^{\text {th }}$ IEEE Real-Time Systems Symposium, 1999, pp. 90-101.

[12] Chen, Ya-Shu, and L.P. Chang, "A real-time configurable synchronization protocol for self-suspending process sets”, Real-Time Systems, Vol. 42, No. 1-3, (2009), pp. 34-62. 
[13] Benini, Luca, D. Bertozzi, A. Guerri and M. Milano, "Allocation, scheduling and voltage scaling on energy aware mpsocs", Integration of AI and OR Techniques in Constraint Programming for Combinatorial Optimization Problems, 2006, pp.44-58.

[14] Kim, Minyoung, S. Banerjee, N. Dutt and N. Venkatasubramanian, "Design space exploration of realtime multi-media MPSoCs with heterogeneous scheduling policies", $4^{\text {th }}$ International Conference Hardware/Software Codesign and System Synthesis, 2006. pp. 16-21.

[15] Kuo, Chin-Fu and Y.C. Hai, "Real-time task scheduling on heterogeneous two-processor systems", Algorithms and Architectures for Parallel Processing, Springer Berlin Heidelberg, 2010, pp. 68-78.

[16] Raravi, Gurulingesh, B. Andersson and K. Bletsas, "Assigning real-time tasks on heterogeneous multiprocessors with two unrelated types of processors", Real-Time Systems, Vol. 49, No. 1, (2013), pp. 29-72.

[17] Instruments, Texas. Inc., "DSP/BIOS II Timing Benchmarks on the TMS320C54x DSP", Technical report, Texas Instruments, http://focus. ti. com, 2000.

[18] Hsieh, Kun-Yuan, Y.C. Lin, C.C. Huang and J.K. Lee, "Enhancing microkernel performance on VLIW DSP processors via multiset context switch", Journal of Signal Processing Systems, Vol. 51, No. 3, (2008), pp. 257-268.

[19] David, M. Francis, J.C. Carlyle and R.H. Campbell, "Context switch overheads for Linux on ARM platforms", Workshop on Experimental computer science, ACM, 2007, pp.3.

[20] Rosen, Jakob, P. Eles, Z. Peng and A. Andrei, "Predictable worst-case execution time analysis for multiprocessor systems-on-chip", 2011 Sixth IEEE International Symposium on Electronic Design, Test and Application (DELTA), pp. 99-104.

[21] Steffens, Liesbeth, M. Agarwal and P. Wolf, "Real-time analysis for memory access in media processing SoCs: A practical approach", Euro micro Conference on Real-Time Systems, 2008, ECRTS'08, pp. 255-265. 
International Journal of Control and Automation Vol.8, No.10 (2015) 